\title{
Assay Type Detection Using Advanced Machine Learning Algorithms
}

\author{
Marzia Hoque Tania \\ Medical Technology Research Centre, \\ Faculty of Science and Engineering \& \\ Faculty of Health, Education, Medicine \\ and Social Care \\ Anglia Ruskin University \\ Chelmsford, United Kingdom \\ ORCID: 0000-0002-4496-1896
}

\author{
Kamal J. Abu-Hassan \\ Department of Physics \\ University of Bath \\ Bath, United Kingdom \\ kjaah20@bath.ac.uk
}

\author{
Khin T. Lwin \\ School of Computing and Digital \\ Technology \\ Teesside University \\ Middlesbrough, United Kingdom \\ ORCID: 0000-0001-8991-3890 \\ M. Shamim Kaiser, SrMIEEE \\ Institute of Information Technology \\ Jahangirnagar University \\ Savar, Bangladesh \\ ORCID: 0000-0002-4604-5461
}

\author{
Antesar M. Shabut \\ School of Arts and Communication \\ Leeds Trinity University \\ Leeds, United Kingdom \\ ORCID: 0000-0001-7219-5685 \\ M. A. Hossain, MIEEE \\ School of Computing and Digital \\ Technology \\ Teesside University \\ Middlesbrough, United Kingdom \\ a.hossain@tees.ac.uk
}

\begin{abstract}
The colourimetric analysis has been used in diversified fields for years. This paper provides a unique overview of colourimetric tests from the perspective of computer vision by describing different aspects of a colourimetric test in the context of image processing, followed by an investigation into the development of a colorimetric assay type detection system using advanced machine learning algorithms. To the best of our knowledge, this is the first attempt to define colourimetric assay types from the eyes of a machine and perform any colorimetric test using deep learning. This investigation utilizes the state-of-the-art pre-trained models of Convolutional Neural Network (CNN) to perform the assay type detection of an enzyme-linked immunosorbent assay (ELISA) and lateral flow assay (LFA). The ELISA dataset contains images of both positive and negative samples, prepared for the plasmonic ELISA based TB-antigen specific antibody detection. The LFA dataset contains images of the universal $\mathrm{pH}$ indicator paper of eight $\mathrm{pH}$ levels. It is noted that the pre-trained models offered $100 \%$ accurate visual recognition for the assay type detection. Such detection can assist novice users to initiate a colorimetric test using his/her personal digital devices. The assay type detection can also aid in calibrating an image-based colorimetric classification.
\end{abstract}

Keywords - computer vision, deep learning, transfer learning, colorimetric test, point-of-care system, diagnosis

\section{INTRODUCTION}

Deep learning has demonstrated incredible success in recent years for image classification including object identification reducing the exasperation of image processing [1]-[4]. Similar to conventional medical image processing, the deep learning techniques [1] are often reluctant towards colors; effectively acting as color-blinds. However, there are some recent developments [5] focusing colorimetry (not to be confused with colorimetric tests) using deep learning.

A colorimetric test provides decisive analysis of the present elements or concentration of the chemical compound facilitated by a color agent. On the other hand, an assay is a mean to investigate the analyte. The point of interest, which could be a substance or chemical constituent that will go through an analytical process, is called an analyte. The assay refers to an analytical process in a number of disciplines to determine the presence of a target entity or quantify the amount or functional activity of the analyte. The colorimetric test such as an LFA facilitates a rapid and easy-touse diagnostic scheme for home-care and resource-limited settings. The focus of this research is such a deliverable health technology at resource-limited settings.

Despite the advantages of deep learning for advance analysis and feature extraction, it is a resource-demanding technology. Therefore, considering the constraints regarding the resource-limited settings and large-scale dataset, this work utilized transfer learning function, where the model is trained on one task and re-used on a second similar task. According to [6], "Transfer learning and domain adaptation refer to the situation where what has been learned in one setting ... is exploited to improve generalisation in another setting." Two most widely used transfer learning approaches are the develop model and the pre-trained model. In the case of a develop model approach, one would require an enormous amount of data while selecting the source-task. On the other hand, the success of the ongoing ImageNet project [1], trained on over 14 million images, has opened the door for faster implementation of deep learning on a smaller dataset using the state-of-the-art pre-trained models to classify images through magnificent features.

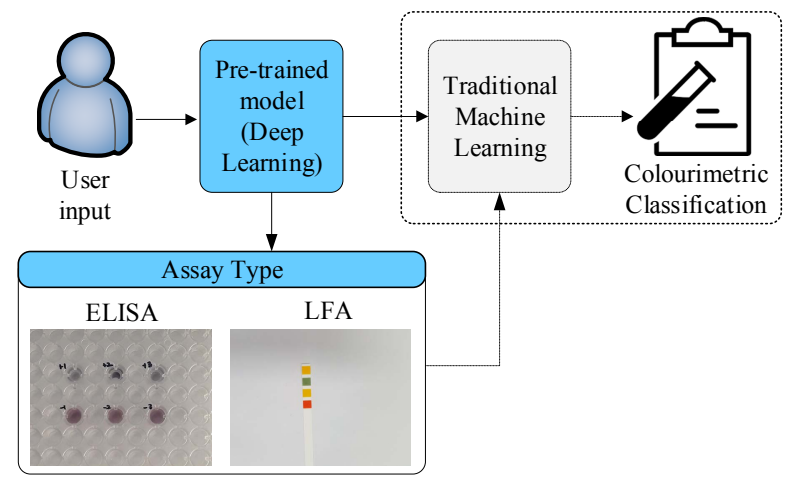

Fig. 1. Comprehensive framework of an image-based colorimetric test. The components in the dotted rectangle were not implemented in this study.

In this work, the pre-trained models are explored for the task of assay type detection (Fig. 1). This paper critically investigates the different form of colorimetric tests from the eyes of machines and presents a proof-of-concept of deep learning-based assay type detection utilizing two assays. 


\section{Colourimetric Assay TyPes}

Conventionally, the assay types can be defined in many ways, such as based on time point, a number of analytes, signal amplification method, type of the substrate and format of the result [7]-[12]. Regarding the detection method, this work considers the assay involving the visible color spectrum only. This section explores the assay types from the computer vision and machine learning (both traditional and advanced) perspective and how it relates to the conventional nature of the assay process.

\section{A. State of Matter}

Based on the state of the matter, the colourimetric assays can be dry as well as wet chemicals based. Both of these assays are well utilized in the reported articles [13]-[18]. The state of matter is an important consideration because, based on the critical review conducted in this paper, the physical state of the sample can dictate the overall system regarding image processing algorithm and feature-set [10], [17], [19].

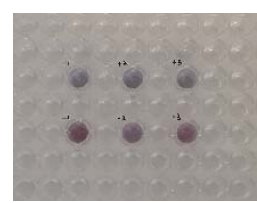

(a)

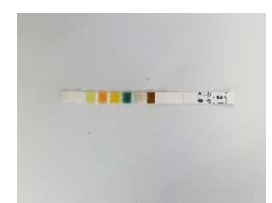

(b)

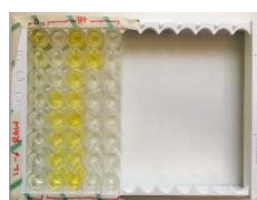

(c)
Fig. 2. Example of assay types. (a) Plasmonic ELISA for TB-antigen specific antibody detection, (b) Urine dipstick, (c) ELISA for analyzing proinflammatory protein production (IL-6)

In Fig. 2, (a) and (c) are wet-chemical-based ELISA images. It can be heavily affected by the ceiling light, which will act as noise during image processing. Spotxel® Reader, a commercial mobile application on the quantitative colourimetric test, has highlighted this particular concern for wet-chemical-based assays [20]. Conceptually, it can be well understood that liquid surface can easily be affected by surrounding light reflectance. Fig. 2(b) is an image of a drychemical based LFA. Although the ambient condition should less affect the dry-chemical based assays, many of the recent works have utilized additional hardware attachments [21], [22].

If the wet samples are placed in a transparent or translucent plate, the image of the assay would also have to deal with the shadow of the colors spread across the empty space of the plate. [23] referred to this issue as the smearing effect.

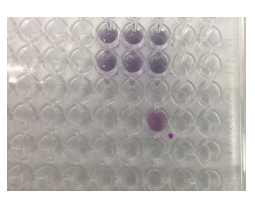

(a)

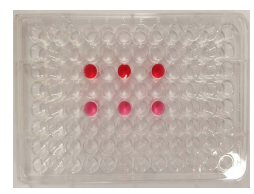

(b)

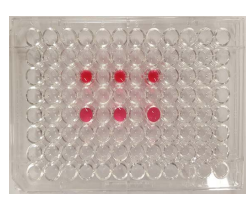

(c)
Fig. 3. Variation in shape of the container. (a) TB-test in a flat plate, (b) Food color in a flat plate, (c) Food color in a round plate

The wet-chemical-based assay can be subject to the shape of the container, i.e. well. Based on the biochemical materials, it can also be a subject to viscosity. In Fig. 3, samples are placed in assay plates varying in well shape.
In Fig. 3 (a), TB-test samples are placed in a plate, where the wells have a flat bottom. For better demonstration, natural food colors were placed in the same variety of assay plate in Fig. 3 (b). The samples in Fig. 3 (a) and (b) vary in viscosity. Intentionally, a minor variation was created within the food coloring as well. The variation is apparent from two rows of Fig. 3 (b). The viscosity and how the chemicals, for example in Fig. 3 (a), bind in different well shape might be an essential consideration in biochemistry, our research did not find any reported article using the computer-aided system to raise any concern regarding these issues. Besides, an advanced computer-aided system should be capable of handling these issues while processing the images. Therefore, this work assumes a negligible impact of the viscosity; the shape of the container may have a higher impact on the imaging than the viscosity.

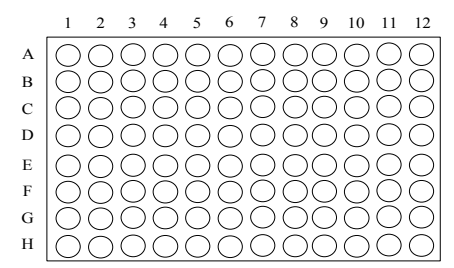

(a)

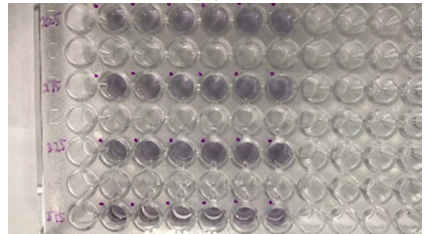

(c)

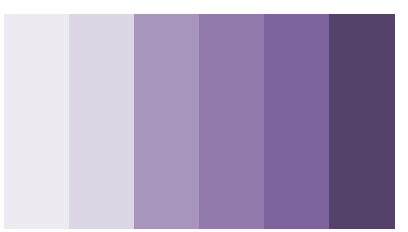

(b)

(d)

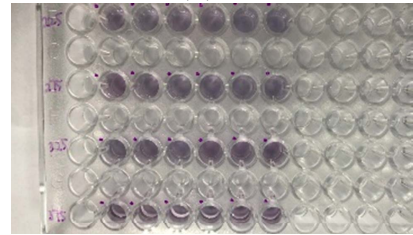

Fig. 4. Visualization of the impact of color concentration. (a) Schematic diagram of an assay plate, (b) Variation of the brightness of the same color or chroma, (c) Sample with light color concentration, (d) Sample with bright color concentration

On the other hand, by observing the images in the reported articles [16], [24] for various types of assay, this work assumes that, despite the materials used, the color formation and brightness of the chroma component (Fig. 4) of a sample can play a vital role in a computer-aided colourimetric decision.

In both Fig. 4 (c) and (d), the concentration of hydrogen peroxide and gold ions were varied in A to $\mathrm{H}$ direction (see Fig. 4 (a)) of the assay plate [18]. The difference between Fig. 4 (c) and (d) is the brightness in color or color concentration. This research assumes that the issue of color concentration can be more crucial for a computer-aided quantitative assay without any hardware attachment.

The state of matter within the context is closely associated with the imaging system, including image acquisition, as well. Image acquisition is an essential step in an image-based system. The early-stage and conventional studies often acquired images by a flatbed scanner [25]-[28]. Images acquired by such scanning involuntarily solves the issue with lighting condition and camera to sample position. However, flatbed scanning is not suitable for wet-chemical-based assays. This paper assumes the assays which can be analysed 
from the images acquired by flatbed scanners can also be analysed from the images captured by mobile phones.

The imaging condition can undoubtedly influence the image quality as well as colors. A wide range of smartphone camera specifications along with intrinsic and extrinsic parameters has been explored in the literature such as the International Organization for Standardization (ISO), flash and focus. Such explorations are exemplified in TABLE I.

TABLE I. Image Acquisition Systems for Colorimetric Tests

\begin{tabular}{|c|c|c|}
\hline Premise & Parameter & Specifics \\
\hline \multirow[t]{3}{*}{$\begin{array}{l}\text { Camera } \\
\text { parameter }\end{array}$} & $\begin{array}{l}\text { Resolution } \\
\text { (MP) }\end{array}$ & $\begin{array}{l}\text { Low: } 3.2[29] \\
\text { High: } 20.7[21]\end{array}$ \\
\hline & ISO & $\begin{array}{l}\text { Varying from } 50[13] \text { to } \\
800[30],[31] \\
\text { Auto [17], [30] }\end{array}$ \\
\hline & $\begin{array}{l}\text { Other } \\
\text { parameters }\end{array}$ & $\begin{array}{l}\text { Constant [10] } \\
\text { Auto [11], [17] }\end{array}$ \\
\hline \multirow[t]{2}{*}{$\begin{array}{l}\text { Camera to } \\
\text { sample position }\end{array}$} & Distance & $\begin{array}{l}\text { Low: } 5 \mathrm{~cm}[32] \\
\text { High: } 2 \text { feet [22] }\end{array}$ \\
\hline & $\begin{array}{l}\text { Exposure } \\
\text { (Angle) }\end{array}$ & $\begin{array}{l}\text { Parallel [13], [17] } \\
\text { Tilted [30] }\end{array}$ \\
\hline
\end{tabular}

Our research assumes, due to the advancement of hardware as well as software components of the phone camera, these parameters can be set to the automatic mode. For field operation, the systems would be exposed to the ambient illumination condition to capture images. Day by day in future, with an advancement of technology, high powered cameras in the mobile devices will be more accessible, and the internal software of the phone camera will be more capable of adjusting the camera parameters according to the ambient condition.

The image acquisition techniques can influence the gap between training and testing datasets [10], [33]. Therefore, a computer-aided colourimetric test should aim for the requirement of minimum fine-tuning in case of such variations including the variations due to the change of platform. The variation of the illumination condition and inconsistencies due to the device to device variation is a more severe concern for traditional machine learning based colorimetric tests [17]. The advanced machine learning techniques such as CNN based deep learning techniques are capable of handling such variations.

\section{B. Colourimetric Result Evaluation Process}

The conventional method defines the type of the result as the qualitative, semi-quantitative and quantitative assay, which are re-defined in this section for an image-based intelligent system.

\section{1) Binary Classification:}

A qualitative assay determines the presence or absence of a substance. The result obtained from a qualitative test would be in yes or no form. Therefore, it is a binary class classification problem. An example is shown in Fig. 5(a).

Although qualitative assays find widespread applications in the various domain, the most well-known application of this assay would be the pregnancy test strip to detect Human Chorionic Gonadotropin (HCG).

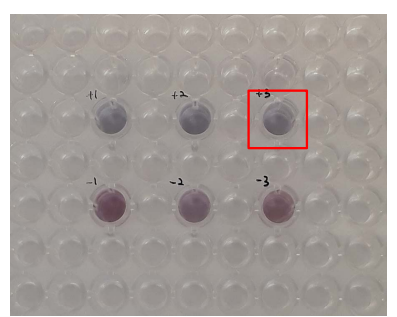

(a)

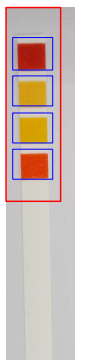

(b)

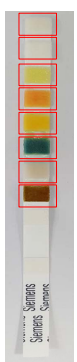

(c)
Fig. 5. Object, target and sample in an image of a colourimetric test. (a) TBtest: image contains six filled wells. In other words, there are six samples. From the computer vision perspective, there are six objects. (b) $\mathrm{pH}$ indicator paper: four objects separated by blue boxes belong to the same $\mathrm{pH}$ test, (c) Multiplex assay: each object is directed to a different target

In theory, the two classes of a qualitative assay should use color opponents. In this way, the assay would provide a naked-eye detection. For a person with no or less medical training, the conventional colourimetric result may be complicated to interpret, for example, the medical patch used by [16]. Therefore, the use of mobile-enabled dengue detection proposed by [16] can support non-medical personnel to understand the presence or absence of dengue virus from the binary classification provided by their system.

\section{2) Multi-class Classification:}

The semi-quantitative assay is in between a qualitative and a quantitative test, yielding an approximation of the quantity or amount of a substance. The result can be compared with a color chart by naked-eye measurement. The clinicians use analyzers such as CLINITEK Status+ Analyzer [34]. This type of test can be seen as a multi-class classification problem as well as a regression problem. An example is shown in Fig. $5(b)$.

Semi-quantitative assays have drawn a lot of attention for computer-aided colourimetric quantification [10], [11], [13], [21]. [10] provided the semi-quantitative colourimetric quantification of fifteen $\mathrm{pH}$ levels (0-14) or classes.

Although the Quantofix Peroxide 100 is supposed to classify only six concentration levels of $\mathrm{H}_{2} \mathrm{O}_{2}(0,1,3,10,30$ and $100 \mathrm{ppm}),[11]$ added five secondary concentration levels $(0.5,2,6.5,20$ and $65 \mathrm{ppm})$ to increase the number of classes. In the absence of an elaborate description, we assume that the images of the test strips containing corresponding colors for these secondary classes can be considered as synthetic data, which may have reliability issue in a real-life scenario.

The research demonstrated in [21] used the paper-based assay to classify five standard concentrations of alcohol as well as nine classes by using intermediate enhanced concentration level. Due to the purpose and nature of the assay, the standard concentration is not an integer value, which is not a concern while performing classification, however, might be a point of interest for more elaborate research while using the intermediate values. Similar to [21], [13] also used class labels of fraction numbers to classify six 
concentration levels of phosphate in water. Nevertheless, the number of classes is related to the choice of the classifier and needs to be considered in the context of the curse of dimensionality [35], [36]. Therefore, care should be given while implementing machine learning algorithms for these colourimetric detections.

\section{3) Regression:}

Quantitative assay helps in determining the amount of a substance, which can be seen as a pure regression problem such as mercury detection in water [24] and tracking HE4 biomarker to detect ovarian cancer [15]. The case studies of this paper mainly focus on the qualitative and semiquantitative colourimetric test due to the scalability of the experiments. Therefore, while reviewing the literature in this paper, more attention is provided towards the classificationbased systems. Conceptually, the image processing framework used for a qualitative and semi-quantitative assay can also be used for a similar quantitative assay. An example of a quantitative assay is shown in Fig. 2(c). In this case, instead of classification, the colourimetric decision has to be produced by regression due to the result-type.

\section{Number of Objects per Sample}

Based on the number of analytes, the assay can be a single target or multiplex. However, this work considers the sample in image format. Therefore, it is essential for the decisionmaking process to realize the number of objects associated with a single sample.

\section{1) Single Object per Sample:}

In Fig. 5, single samples are outlined with a red box. In Fig. 5 (a) and (b), the single object or well represents a single sample, whereas in Fig. 5(c), although the assay is dipped into a single sample, each red box belongs to a single test (which can be interpreted as a single sample from a different perspective). In Fig. 5(a), single sample implies sputum of an individual and single test indicates plasmonic ELISA for TBantigen specific antibody detection.

In the literature, most of the work considers the assay as a single object/ sample [11], [21]. [21] used paper test strips [37] capable of measuring a single concentration using a single color pad. [11] used paper strips to quantify $\mathrm{H}_{2} \mathrm{O}_{2}$ using test strips of Quantofix Peroxide 100. This test strip [11] can provide a semi-quantitative measurement of $\mathrm{H}_{2} \mathrm{O}_{2}$ using a single color pad. Both of these assays [11], [21] are for rapid test reading.

Despite being a single object/ sample kind of assay, an assay can hold multiple samples. For an image of a 96-well plate, there can be multiple samples (maximum 96 samples). The assay types are shown in Fig. 5 (a) and Fig. 2(c). There are mobile applications available in the commercial appstores which can act as a plate reader such as AssayColor [38] and Spotxel ${ }^{\circledR}$ Reader [20]. Both of these mobile applications can process multiple samples in an assay plate, treating each sample as a single object. However, these mobile applications utilize virtual plate to deal with a 96 -well plate.
2) Multiple Objects per Sample:

In Fig. 5(b), multiple objects belong to a single sample as well as a single test, i.e. $\mathrm{pH} 3$ and are collectively responsible for producing a decision. If these objects are to be detected individually (outlined in blue boxes), then the feature-set would require to be multiplied by the number of objects/ sample. Although the feature-set is going to be elongated, it is not logical to consider all four color chambers as one object because it would contradict the purpose of the assay type.

Multiple objects per sample type of assay is evident in the literature as well. [16] used a mobile-enabled paper-based squared-shape medical patch containing four wells which were the objects to be detected. The assay may cost only 20 cents approximately. A droplet of blood from a patient would be required to come in to contact with the patch so that the blood can be diffused into the wells, i.e. different chamber of the patch. The approximate reaction time was 30 minutes. Based on the analytes in the blood, the color of the wells were transformed. Now, each of these wells belongs to the same test, i.e. dengue detection and all four of these wells or objects were collectively responsible for providing this test-decision.

[10] utilized $\mathrm{pH}$ strips containing multiple color pads or objects which can be considered as another example of multiple objects per sample to perform a single sample-test. However, no description is provided by [10] regarding these objects detection mechanism. The method described in [33] can also be considered under this category. [33] required to capture the image of both 'control' and 'test' paper strip, hence involved multiple objects.

\section{3) Multi-test per Image and Single Object per} Test:

The multiplex assays are capable of performing multiple tests on a single sample, e.g. urine of an individual. Therefore, each object, block or color pad in Fig. 5(c) represents different test type such as glucose, $\mathrm{pH}$, protein and ketone.

In the literature, [39] proposed a chemically patterned microfluidic paper-based analytical device for heavy metal detection. Their dry-chemical based multiplex colourimetric system is comprised of five color chambers, which can be considered as five blobs or objects that can assist in determining nickel, chromium and mercury. However, the system did not include any computer-aided scheme.

[31] proposed a mobile-enabled paper-based microfluidic device for $\mathrm{pH}$ and nitrite detection. In their assay, there are four chambers for $\mathrm{pH}$, three for nitrite and one for reference. In order to precisely detect these all eight objects, they used two additional blobs to ease the object identification process.

Based on the aforementioned number of objects in the sample, the computerized systems in the literature utilize virtual overlay mostly for 96-well plates [20], [40]. Even the overlays for 96-well plates, the available systems assume equal distances among the wells such as [20], [40], which may not be the case in reality. Therefore, this work proposes a virtual overlay based on the type of the assay and shape of 
the objects, where the position of the objects are user-defined and modifiable based on user-interaction (Fig. 6).

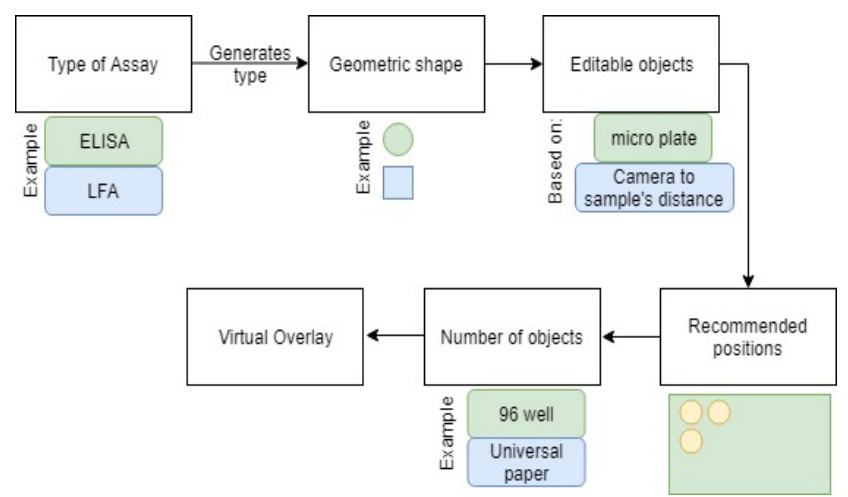

Fig. 6. Recommended virtual overlay based on the type of the assay

In Fig. 6, the assay type can be detected using inductive transfer as illustrated in Fig. 1. Based on the type of the assay, the rest of the recommended system in Fig. 6 can be generated. The shape of the objects would be defined by the assay-type. There is a massive number of possible variations, the objects need to be modifiable. If the user is given guided partial flexibility to move a few objects, then the rest of the objects can be aligned automatically. In this way, the user would be given more flexibility to choose the assay brand and imaging parameters such as the camera to sample position. Such an interactive overlay could ease the necessity to use computational intelligence to separate the region of interest. Alternatively, one could use computer vision and machine learning techniques such as [17], [41].

\section{METHODS}

\section{A. Data Collection}

This paper includes a balanced dataset consisting of 1600 images with an equal proportion of two types of assay (Fig. 1). One is the Gold Nanoparticles (AuNP) based plasmonic ELISA for TB-antigen specific antibody detection, referred to as the TB-test in this paper. The description of the sample preparation for the TB-test is provided in [9], [41], [42]. However, development of the biosensor is not the focus of this work. In terms of the state of matter, this is wet-chemical based sandwich-ELISA assay. The assay is sensitive to temperature and geo-location, and has single objects per sample but multiple samples per image.

The other assay type utilized in this paper is the $\mathrm{pH}$ indicator universal test strips, ranging from 3.0 to 10.0 . It is a widely used dry-chemical based LFA, which is stable at room temperature and suitable for field operations as well, and has multiple objects per sample. This assay can be further subcategorized for multi-class classification within the assay type.

Both of these assay types are visually different in terms of size, shape and color. These assays are also different due to the materials and functionality. The images of both assay types were captured via a number of $\mathrm{iOS}$ and Android-based mobile devices, and illumination condition also varied greatly to create a robust dataset.

\section{B. Pre-trained Models}

In this paper, the state-of-the-art pre-trained models for visual recognition problems were investigated to recognize the assay type utilizing a Windows 10 Intel ${ }^{\circledR}$ Core ${ }^{\mathrm{TM}}$ i7-4470 $\mathrm{CPU}$ at $3.40 \mathrm{GHz}, 16 \mathrm{~GB}$ and 64 with bit $\mathrm{OS}$ machine to train these models using MATLAB 2018a. When there is a substantial amount of large data and similarity between the tasks is also high, the pre-trained models are supposed to exhibit good performance.

Among the best performing pre-trained model of ImageNet challenge [1], this paper considered AlexNet [2] and Inception v-3 [3] to provide a binary classification of the assay type due to the structure of these models. The AlexNet employed a series network for deep learning, where all the layers are arranged one after the other. Among the 25 layers within the network, there is a single input layer, eight layers with learnable weights consisting convolutional layers and fully connected layers, and a single output layer, whereas the Inception v-3 is based on a directed acyclic graph (DAG) network.

We have utilized pre-trained models in this work, and these pre-trained weights are supposed to be better than randomly initialized weights. Therefore, one should aim at retaining the essence of the traits of the original models, so that the pre-trained weights are not highly altered instantly. Therefore, the common practice is to use the initial learning rate ten times smaller than the actual model.

The solver training network of the AlexNet utilized stochastic gradient descent with momentum (SDGM) as Equation 1 [43]:

$$
\theta_{l+1}=\theta_{l}-\alpha \nabla E\left(\theta_{l}\right)+\gamma\left(\theta_{l}-\theta_{l-1}\right)
$$

where $\theta=$ parameter vector, $l=$ iteration number, $E(\theta)=$ cost function, initial learning rate $\alpha=0.001$ and momentum $\gamma=0.9$. The maximum number of epochs were 20 and the minibatch size was 64 . On the other hand, there were six epochs with a mini-batch size of 10 while using the 42-layer deep inception, and the maximum iteration was 672 . The learning rate was 0.0001 . In this case, the weights of the initial layer of the module were chosen to be frozen. The Inception module utilized the same iterative method for optimizing the differentiable objective function, i.e. SDGM.

\section{RESULTS}

\section{A. Assay Type Detection in the Desktop Environment}

This paper involves only two assay types (Fig. 1). Replacing the final layers of the pre-trained models with our particular problem to fine-tune the models, both AlexNet and Inception provided $100 \%$ accuracy (Fig. 7). For both cases, the specificity and sensitivity were found to be $99.54-100 \%$ (using 95\% confidence interval).

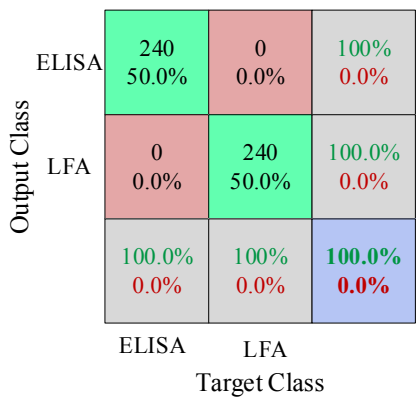

Fig. 7. Confusion matrix of the testing dataset of both AlexNet and Inception

The final layers of AlexNet had only two fully connected layers to detect the assay type. The training on 1,600 images 
took 15 min 42 seconds on a single CPU, whereas the prediction was provided within a fraction of a second (TABLE II).

TABLE II. Comparative performance of Alexnet and inception for assay type detection

\begin{tabular}{|l|l|l|}
\hline Model & AlexNet & Inception \\
\hline Accuracy (\%) & 100 & 100 \\
\hline $\begin{array}{l}\text { Training time } \\
\text { (mm:ss) }\end{array}$ & $15: 42$ & $349: 31$ \\
\hline Network & Series & DAG \\
\hline $\begin{array}{l}\text { Memory } \\
\text { Occupancy (MB) }\end{array}$ & 621 & 232 \\
\hline
\end{tabular}

In the case of Inception, the training time was considerably longer than the AlexNet. The Inception also possesses a higher network complexity. However, Inception has several advantages over AlexNet such as batch normalization and image distortion. Moreover, due to the network structure performing several small convolutions to drastically reduce the number of parameters, the memory occupancy of Inception was downsized.

\section{B. Assay Type Detection on the Mobile Platform}

The above mentioned trained models are heavy for mobile devices, however, can be easily uploaded to a server. To provide a prediction of the assay type for an entirely unseen image, a third party application was utilized to capture the new image on-site by MATLAB mobile. The server takes less than a minute to be connected with the mobile phone, depending on the specification of the mobile device, server and internet connection. The prediction time on the mobile platform was slightly lower than the desktop application when the trained model was uploaded to the MATLAB server, which supports the findings regarding the mobile-enabled server connected colourimetric test by [16], [21].

\section{DISCUSSION}

From the aforementioned results, it can be well perceived that the pre-trained models are capable of predicting the accurate assay type using a reasonable dataset $(<1000$ samples per class) because the similarity of the task is high, i.e. visual recognition. However, when it comes to the final colorimetric classification, this work explored two alternative approaches.

\section{A. Colorimetric Classification using Deep Learning}

The deep learning techniques presented in this paper using inductive transfer possess the restricted capability to detect colors in the absence of any variation in the geometric features.

To support our claim, we extended our study to perform colorimetric classification of $\mathrm{pH}$ levels (ranging from 3.0 to 8.0) from the LFA type. The class being the assay type, where LFA represents a singular class, let us refer to this $\mathrm{pH}$ level classification as the intra-class classification. This intra-class classification is the colorimetric detection, for which we extended the dataset to include 1000 images per class.

Similar to the assay type detection, the final layers of AlexNet was replaced to fine tune the model with the current problem (Fig. 8). Training the AlexNet using the same solver training network specification as described earlier, the classification accuracy was found to be significantly poor. There were 87 iterations for each of 20 epochs with a constant learning rate of 0.001 . The overall accuracy of the model was $\sim 93 \%$. The convergence can be visualized in Fig. 9.

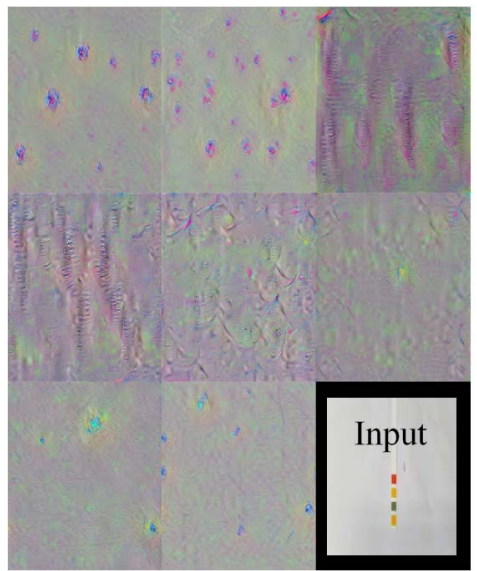

Fig. 8. Visualization of features at final fully connected layer of AlexNet for intra-class classification

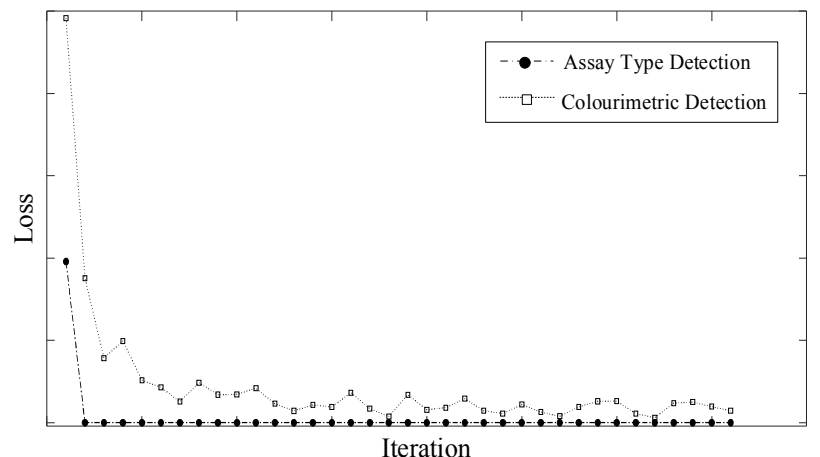

Fig. 9. Convergence of AlexNet for assay type detection and colorimetric detection

The general perception regarding deep learning is- larger dataset produces a better result. From the critical analysis of the features of each layer of AlexNet and the training progress, it can be perceived that the poor classification accuracy for the intra-class classification could be due to the insufficient number of samples. The performance of Inception was found to be worse than the AlexNet. Hence, the result obtained by Inception $\mathrm{v}-3$ is not discussed for the colorimetric classification.

Using 8000 images, the pre-trained model of AlexNet, consisting series network, was trained by taking a considerable amount of time. The elapsed time was 76 minutes and 27 seconds. However, the number of iterations and epoch were inadequate to provide an accurate colorimetric classification for the $\mathrm{pH}$ levels. The convergence rate (Fig. 9) indicates a longer training cycle may improve the performance of the network, which would also expose the model to the risk of overfitting problem. The need of a significantly large dataset and longer training cycle would effectively require more enhanced resources, e.g. higher computational power, which contradicts with our research goal. 


\section{B. Comprehensive Framework for Colorimetric Classification}

Based on the above discussion, this work proposes (Fig. 1) to utilize traditional machine learning technique to reduce complexity of the image-based system and limit the requirement of large-scale dataset as well as resources. As mentioned earlier, the pre-trained models are useful when the task is similar. The colourimetric test of a specific assay would have only one dissimilarity, i.e. color. Therefore, it is not logical to use the deep neural network for such intra-class classification, especially when one of our goals is resourcelimited settings. The results also indicate that these pretrained models are more suitable for assay type detection rather than binary or multi-class classification within the assay type. The geometric shape of assay and location of the colored samples are easily distinguishable for these pretrained models using the standard kernels with a reasonable amount of dataset. However, the intra-class classification would require the model to search for only the colors even when the rest of the geometric features are similar, and occurrence of those colors are in the same location. Therefore, it is more rational to use a simpler machine learning model for the colorimetric classification (such as [17], [41]), instead of building more deep layers which would require more processing capacity, memory size, larger dataset and more dependency on the cloud-based approach [11], [21].

An image-based colourimetric test often requires calibration and to recognize the defined color chambers [32], [44]; the lighting condition is another paramount challenge [11]. Moreover, the brand to brand variation within the assay type could be discouraging to users at home-care settings. The advance machine learning techniques using inductive transfer can assist in addressing these concerns. The variation regarding size, shape and color of the assay by different commercial brands can also be mitigated to certain extent through these deep neural networks (Fig. 2).

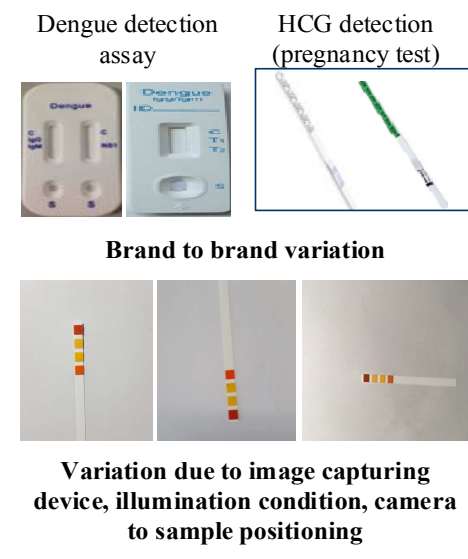

Fig. 10. Purpose of assay type detection using deep learning

Few examples of brand to brand variation is provided in the first row (Fig. 10). The use of similar assays using imagebased scheme can be found in [45]. Deep learning techniques are also capable to deal with many invariances, including illumination, as illustrated in the bottom row of Fig. 10.

\section{CONCLUSION}

In this paper, we have redefined colorimetric tests from the imaging context for the convenience of future researchers to develop and deploy an image-based intelligent system for colorimetric tests. We have also presented deep learning based assay type detection to support an intelligent image-based colorimetric test framework (Fig. 1) for future mobile enabled diagnosis. Both 8-layers deep AlexNet consisting series network and 42-layers deep DAG network of Inception v-3 showed $100 \%$ accuracy to detect the assay type in real time on the mobile platform. The training time of Inception was significantly higher than AlexNet. However, from the users' point of view, only the prediction time is essential as the users will not require to consider the training time for an already trained model.

Based on the model complexity, AlexNet is more preferable than Inception for our task. On the other hand, the size of the trained model was found to be much smaller for Inception. Therefore, in terms of memory occupancy, Inception is a better choice to identify the type of the assay, which can support a naïve user to begin a colorimetric test and deal with the variation between different commercial brands for the same assay type. It can also simplify an intelligent colorimetric test to mitigate the challenges due to lighting environment, because these advanced machine learning techniques can handle the invariances in images.

With the aid of a larger dataset and a high performing computational system, our future research will include an empirical study using the developed model approach, instead of using pre-trained models using inductive transfer learning to evaluate the possibility of a better performance while using deep learning techniques to produce a colorimetric decision.

\section{ACKNOWLEDGMENT}

The research work has been funded by Erasmus Mundus Partnerships Action 2 "FUSION" (Featured eUrope and South asIa mObility Network). Grant reference number: 2013-3254 1/001001. The authors' thank Prof Nor Azah Yusof and her team, Universiti Putra Malaysia for their support to collect the ELISA dataset. The original ELISA dataset was generated as part of the project named 'TB-Test - A smart mobile enabled scheme for tuberculosis testing', supported by British Council Newton Institutional Links and Newton-Ungku Omar Fund (Grant ID: 216385726), The authors also thank Dr Mohammad Najlah and Mr Paul Cotton, Anglia Ruskin University for their support to conduct the laboratory experiments on LFA.

\section{REFERENCES}

ImageNet, "ImageNet," 2016. [Online]. Available: http://imagenet.org/index. [Accessed: 28-Jul-2018].

A. Krizhevsky, I. Sutskever, and G. E. Hinton, "ImageNet Classification with Deep Convolutional Neural Networks," in Advances in neural information processing systems, 2012, pp. 1097-1105.

[3] C. Szegedy, V. Vanhoucke, S. Ioffe, J. Shlens, and Z. Wojna, "Rethinking the Inception Architecture for Computer Vision," in IEEE Conference on Computer Vision and Pattern Recognition (CVPR), 2016.

[4] S. Khan, N. Islam, Z. Jan, I. U. Din, and J. J. P. C. Rodrigues, “A novel deep learning based framework for the detection and classification of breast cancer using transfer learning," Pattern Recognit. Lett., vol. 125, no. July, pp. 1-6, 2019.

[5] M. Kerkech, A. Hafiane, and R. Canals, "Deep leaning approach with colorimetric spaces and vegetation indices for vine diseases 
detection in UAV images," Comput. Electron. Agric., vol. 155, pp. 237-243, Dec. 2018

[6] I. Goodfellow, Y. Bengio, and A. Courville, Deep Learning. MIT Press, 2016.

[7] M. H. Tania, K. T. Lwin, A. M. Shabut, and M. A. Hossain, "Clustering and Classification of a Qualitative Colorimetric Test," in 2018 International Conference on Computing, Electronics \& Communications Engineering (iCCECE), 2018, pp. 7-11.

[8] M. S. Khan and G. Garnier, "Direct measurement of alkaline phosphatase kinetics on bioactive paper," Chem. Eng. Sci., vol. 87 , pp. 91-99, Jan. 2013.

[9] N. M. Bakhori, N. A. Yusof, J. Abdullah, and H. Wasoh, "Immuno Nanosensor for Ultrasensitive and Affordable Naked Eye Detection of Tuberculosis," Sensors, vol. 18, no. 6, pp. 1-10, 2018.

[10] A. Y. Mutlu, V. Kılıc, G. K. Özdemir, A. Bayram, N. Horzum, and M. E. Solmaz, "Smartphone-based colorimetric detection via machine learning," Analyst, vol. 142, no. 13, pp. 2434-2441, 2017.

[11] M. E. Solmaz, A. Y. Mutlu, G. Alankus, V. Kılıç, A. Bayram, and N. Horzum, "Quantifying colorimetric tests using a smartphone app based on machine learning classifiers," Sensors Actuators $B$ Chem., vol. 255, pp. 1967-1973, Feb. 2018.

[12] A. K. Yetisen, "Holographic Sensors," 2014.

[13] G. Alankus, N. Horzum, A. Y. Mutlu, A. Bayram, and M. E. Solmaz, "Single-Image-Referenced Colorimetric Water Quality Detection Using a Smartphone," ACS Omega, vol. 3, no. 5, pp. 5531-5536, 2018.

[14] C. Sicard et al., "Tools for water quality monitoring and mapping using paper-based sensors and cell phones," Water Res., vol. 70, pp. 360-369, 2015

[15] G. G. Lin and J. G. Scott, "Integration of cell phone imaging with microchip ELISA to detect ovarian cancer HE4 biomarker in urine at the point-of-care," Lab Chip, vol. 100, no. 2, pp. 130-134, 2012.

[16] J. Matthews, R. Kulkarni, M. Gerla, and T. Massey, "Rapid dengue and outbreak detection with mobile systems and social networks," Mob. Networks Appl., vol. 17, no. 2, pp. 178-191, 2012.

[17] A. M. Shabut et al., "An intelligent mobile-enabled expert system for tuberculosis disease diagnosis in real time," Expert Syst. Appl., vol. 114, pp. 65-77, Jul. 2018.

[18] M. H. Tania, K. T. Lwin, K. Abuhassan, and N. M. Bakhori, "An Automated Colourimetric Test by Computational Chromaticity Analysis: A Case Study of Tuberculosis Test," in Advances in Intelligent Systems and Computing, 2017, vol. 616, no. 2014, pp. 313-320.

[19] J. E. Smith, D. K. Griffin, J. K. Leny, J. a Hagen, J. L. Chávez, and N. Kelley-Loughnane, "Colorimetric detection with aptamer-gold nanoparticle conjugates coupled to an android-based color analysis application for use in the field," Talanta, vol. 121, pp. 247-55, 2014.

[20] Sicasys Software GmbH, "Spotxel® Reader,” Google Play, 2017. [Online]. Available: https://play.google.com/store/apps/details?id=com.sicasys.spotxel $\& \mathrm{hl}=\mathrm{en}$. [Accessed: 12-Jan-2018].

[21] H. Kim, O. Awofeso, S. Choi, Y. Jung, and E. Bae, "Colorimetric analysis of saliva--alcohol test strips by smartphone-based instruments using machine-learning algorithms," Appl. Opt., vol. 56, no. 1, pp. 84-92, 2017.

[22] S. Feng, R. Caire, B. Cortazar, M. Turan, A. Wong, and A. Ozcan, "Immunochromatographic diagnostic test analysis using google glass," ACS Nano, vol. 8, no. 3. pp. 3069-3079, 2014.

[23] Sicasys Software GmbH, "Smartphone-based Colorimetric Image Reader Quick Start Guide," 2017.

[24] W. Chen, X. Fang, H. Li, H. Cao, and J. Kong, “A Simple PaperBased Colorimetric Device for Rapid Mercury(II) Assay," Sci. Rep., vol. 6, no. August, 2016.

[25] K. S. Suslick, N. A. Rakow, and A. Sen, "Colorimetric senso arrays for molecular recognition," Tetrahedron, vol. 60, no. 49, pp. 11133-11138, 2004.

[26] P. J. Mazzone et al., "Diagnosis of lung cancer by the analysis of exhaled breath with a colorimetric sensor array.," Thorax, vol. 62, no. 7 , pp. 565-8, 2007.

[27] P. J. Mazzone et al., "Exhaled Breath Analysis with a Colorimetric Sensor Array for the Identification and Characterization of Lung Cancer," J Thorac Oncol., vol. 7, no. 1, pp. 137-142, 2013.

[28] X. Zhong et al., "Rapid and Ultrasensitive Detection of Biogenic Amines with Colorimetric Sensor Array," Sensors Actuators B Chem., Jul. 2018.

[29] D. Cooper, B. Callahan, P. Callahan, and L. Burnett, "Mobile Image Ratiometry: A New Method for Instantaneous Analysis of Rapid Test Strips," in Nature Precedings, 2012, pp. 2-3.

[30] H. Karlsen, "Smartphone-based urinary biomarker detection: an application-oriented device and algorithm," University College of Southeast Norway, 2018.

[31] N. Lopez-Ruiz et al., "Smartphone-based simultaneous pH and nitrite colorimetric determination for paper microfluidic devices," Anal. Chem., vol. 86, no. 19, pp. 9554-9562, 2014.

[32] A. K. Yetisen, J. L. Martinez-Hurtado, A. Garcia-Melendrez, F. da Cruz Vasconcellos, and C. R. Lowe, "A smartphone algorithm with inter-phone repeatability for the analysis of colorimetric tests," Sensors Actuators B Chem., vol. 196, pp. 156-160, 2014.

[33] C. Sicard et al., "Supplimentary Document: Tools for water quality monitoring and mapping using paper-based sensors and cell phones," Water Research, vol. 70. pp. 360-369, 2015.

[34] Siemens Healthcare GmbH, "CLINITEK Status+ Analyzer," 2018. [Online]. Available: https://www.healthcare.siemens.com/urinalysis-

products/urinalysis-systems/clinitek-status-analyzer. [Accessed: 19-May-2018].

[35] R. E. Bellman, "Adaptive Control Processes: A Guided Tour," Princeton University Press, 1961.

[36] R. E. Bellman, Dynamic programming, Republishe. Courier Dover Publications, 2003.

[37] Chematics Inc., "Alco-Screen test strips," 2018. [Online]. Available: https://chematics.com/. [Accessed: 18-Sep-2018].

[38] Alidans srl, "AssayColor," Android App on Google Play, 2015. [Online]. Available: https://play.google.com/store/apps/details?id=com.alidans.assayc olor. [Accessed: 10-Jan-2017]

[39] J. P. Devadhasan and J. Kim, "A chemically functionalized paperbased microfluidic platform for multiplex heavy metal detection," Sensors Actuators B Chem., vol. 273, pp. 18-24, Nov. 2018.

[40] S. K. Vashist, T. van Oordt, E. M. Schneider, R. Zengerle, F. von Stetten, and J. H. T. Luong, "A smartphone-based colorimetric reader for bioanalytical applications using the screen-based bottom illumination provided by gadgets," Biosens. Bioelectron., vol. 67, pp. 248-255, 2015

[41] K. J. Abuhassan et al., "Automatic Diagnosis of Tuberculosis Disease Based on Plasmonic ELISA and Color-based Image Classification," in 39th Annual International Conference of the IEEE Engineering in Medicine and Biology Society (EMBC), 2017, pp. 4512-4515.

[42] M. A. H. Marzia Hoque Tania, K. T. Lwin, Kamal AbuHassan, Noremylia Mohd Bakhori, Umi Zulaikha Mohd Azmi, Nor Azah Yusof, "An Automated Colourimetric Test by Computational Chromaticity Analysis: A Case Study of Tuberculosis Test," Adv. Intell. Syst. Comput., vol. 616, pp. 313-320, 2017.

[43] K. P. Murphy, Machine learning: A probabilistic perspective. MIT Press, 2012.

[44] S. Garg, R. S. Ramprasaath, S. Kapur, and K. M. M. Rao, "Automated colorimetric analysis in paper based sensors," in Image Processing (ICIP), 2014 IEEE International Conference on, 2014, pp. 3607-3611.

[45] L. Yu, Z. Z. Shi, C. Fang, Y. Y. Zhang, Y. S. Liu, and C. M. Li, "Disposable lateral flow-through strip for smartphone-camera to quantitatively detect alkaline phosphatase activity in milk," Biosens. Bioelectron., vol. 69, pp. 307-315, 2015. 